\title{
Myocardial contractility and afterload in aortic stenosis
}

\author{
Tazim Merchant ${ }^{1,6^{*}}$, Nathaniel Reichek ${ }^{1,2}$, Eddy Barasch ${ }^{1,2}$, Madhavi Kadiyala ${ }^{1,2}$, Meghana Jayam³ ${ }^{3}$ Simcha Pollack ${ }^{1,4}$, \\ Jie J Cao ${ }^{1,2}$, Alistair Young ${ }^{5}$ \\ From 18th Annual SCMR Scientific Sessions \\ Nice, France. 4-7 February 2015
}

\section{Background}

In aortic stenosis(AS) with normal left ventricular ejection fraction(LV EF), circumferential and longitudinal systolic strains(CSt, LSt) are reduced but the underlying mechanisms are uncertain. Conventional indices of afterload(A) per unit of myocardium are suboptimal for strain analysis and based on inaccurate assumptions about myocardial properties, while conventional contractility $(\mathrm{C})$ indices address LV chamber, not myocardial properties. We have shown that the product of end-systolic pressure $(\mathrm{P})$ and volume $(\mathrm{V})$, divided by $\mathrm{LV}$ mass $(\mathrm{M})$, or $(\mathrm{PV} / \mathrm{M})$, is a more effective index of A than conventional wall stress(Mirsky, J. Biophys. 1969) for strain analysis, while the ratio of strain to $\mathrm{PV} / \mathrm{M}$ is an effective strain-based index of $C$. These methods were used to evaluate the mechanism of reduced LV strain in AS with preserved EF.

\section{Methods}

Age and gender matched patients with AS and normal volunteers $(\mathrm{NL})$ were compared (Table). CMR was performed on a $1.5 \mathrm{~T}$ scanner, using TrueFISP cine short and LV long axis imaging and volumetrics and featuretracking CSt, LSt and strain rates evaluated(CIM, U. of Auckland). A was quantitated as LV end-systolic PV/M, and as circumferential and meridional wall stress(CWS, MWS). $\mathrm{C}$ was assessed using the ratios, $\mathrm{CSt} /(\mathrm{PV} / \mathrm{M}), \mathrm{LSt} /$ (PV/M), CSt/CWS and LSt/MWS.

\section{Results}

(Table 1): Mean EF was normal in both AS and NL but systolic CSt and LSt were reduced in AS, without significantly increased afterload. In contrast, contractility, expressed as $\mathrm{CSt} /(\mathrm{PV} / \mathrm{M})$ and $\mathrm{LSt} /(\mathrm{PV} / \mathrm{M})$ was reduced in $\mathrm{AS}$, although

${ }^{1}$ St. Francis Hospital, Research and Education Foundation, Roslyn, NY, USA Full list of author information is available at the end of the article wall stress based indices and strain rate(SR) were not. There were strong negative correlations between PV/M and EF in AS and NL and strong positive correlations between PV/M based contractile indices and $\mathrm{EF}$, but regression slopes for strain/PV/M vs.EF and versus strain itself were significantly higher in AS, underscoring the role of reduced contractility. In a subset of AS patients $(\mathrm{n}=8)$, extracellular volume data were available but correction for smaller myocyte fraction of LV mass $(72 \%$ AS vs $76 \% \mathrm{NL})$ did not significantly alter results.

Table 1

\begin{tabular}{cccc}
\hline & $\mathrm{NL}$ & $\mathrm{AS}$ & $\mathrm{P}$ \\
\hline AGE(yrs,(s.d.)) & $71.2(10.9)$ & $71.8(10.8)$ & $\mathrm{ns}$ \\
\hline Gender & $8 \mathrm{M}, 7 \mathrm{~F}$ & $8 \mathrm{M}, 7 \mathrm{~F}$ & $\mathrm{~ns}$ \\
\hline EF(\%) & $58(6)$ & $56(11)$ & $\mathrm{ns}$ \\
\hline LVM(g) & $89(23)$ & $137(43)$ & $<0.0001$ \\
\hline LVP(mmHg) & $138(12)$ & $203(27)$ & $<0.0001$ \\
\hline Mean Gradient(mmHg) & $\mathrm{X}$ & $49(18)$ & $\mathrm{X}$ \\
\hline AVA(cm2) & $\mathrm{X}$ & $0.8(0.2)$ & $\mathrm{X}$ \\
\hline Global CStrain(\%) & $-19.7(2.7)$ & $-16.5(4.4)$ & 0.02 \\
\hline Global LStrain(\%) & $-15.6(2.4)$ & $-12.2(3.2)$ & $<0.003$ \\
\hline PV/M(mmHgxml/g) & $91.5(23.9)$ & $98.6(34.8)$ & $\mathrm{ns}$ \\
\hline CWStress (103dyn/cm2) & $228.9(79.3)$ & $202.3(81)$ & $\mathrm{ns}$ \\
\hline MWStress (103dyn/cm2) & $89.6(35.3)$ & $63.9(39.4)$ & $\mathrm{ns}$ \\
\hline CStrain/PV/M(\%/mmHgxml/g) & $-0.24(0.1)$ & $-0.14(0.1)$ & 0.05 \\
\hline LStrain/PV/M(\%/mmHgxml/g) & $-0.18(0.1)$ & $-0.14(0.1)$ & 0.03 \\
\hline CStrain/CWS(\%/103dyn/cm2) & $-0.1(0.04)$ & $-0.1(0.06)$ & $\mathrm{ns}$ \\
\hline LStrain/MWS(\%/103dyn/cm2) & $-0.2((0.01)$ & $-0 / 3(0.06)$ & $\mathrm{ns}$ \\
\hline R PV/M vs EF & -0.82 & -0.82 & $<0.01$ each \\
\hline R CSt/PV/M vs EF & 0.87 & 0.85 & $<0.00001$ each \\
\hline R LSt/PV/M vs EF & 0.82 & 0.84 & $<0.00001$ each \\
\hline
\end{tabular}




\section{Conclusions}

Reduced systolic strain in AS with normal EF is due principally to reduced contractility, presumably due to known alterations in myocardial energetics, calcium handling and contractile proteins in hypertrophied myocardium. In contrast, differences in afterload are modest, so that overt "afterload excess" at the myocardial level is not seen, due to the adequacy of compensatory hypertrophy. In addition, the dependence of myocardial performance on contractile state is more marked in AS than in NL. Afterload and contractile indices based on PV/M were more useful than those based on conventional wall stress estimates.

\section{Funding}

Supported by the St. Francis Research Foundation.

\section{Authors' details}

${ }^{1}$ St. Francis Hospital,Research and Education Foundation, Roslyn, NY, USA.

${ }^{2}$ Medicine, Stony Brook University, Stony Brook, NY, USA. ${ }^{3}$ Biomedical Engineering, University of Pennsylvania, Philadelphia, NY, USA. ${ }^{4}$ Applied Mathematics, St. John's University, New York City, NY, USA. ${ }^{5}$ Anatomy, University of Auckland, Auckland, New Zealand. 'Eocust Valley High School, Locust Valley, NY, USA.

Published: 3 February 2015 